\title{
DISEÑO DE CONTRATOS EN EL SECTOR DE LA SALUD
}

Nebel Silvana Moscoso"

\section{INTRODUCCION}

Actualmente en la Argentina los servicios hospitalarios son brindados, en la mayoría de los casos, por grandes estructuras tanto edilicias como de múltiples servicios. Es decir coexisten considerables inversiones en capital y tecnología como en recursos humanos de diferentes especialidades. En dichas estructuras se combinan tanto el servicio hospitalario como las prácticas ambulatorias y los consultorios externos. La contrapartida económica proviene de los recursos provistos por los pacientes, las obras sociales, las medicinas prepagas y/o el gasto público destinado a la salud.

La oferta de los servicios hospitalarios puede provenir del sector privado o del sector público. En el primer caso el objetivo es, además de brindar los servicios para la atención de la salud, maximizar el beneficio derivado de las prestaciones ofrecidas. En el segundo caso, el objetivo es exclusivamente brindar los servicios para la atención de la salud.

La demanda de estos servicios surge tanto de pacientes con algún seguro de salud (obra social o medicina prepaga) con el que cubren parte de sus gastos como de pacientes sin cobertura que no poseen ningún tipo de seguro y recurren generalmente a los servicios hospitalarios públicos para cubrir sus necesidades del cuidado de la salud.

El objetivo del presente trabajo es mostrar la existencia de asimetrías de información entre los profesionales de la salud (médicos) y los pacientes a través de la relación Agente - Principal. Una vez analizado el conflicto de intereses entre dichas partes, se plantean las necesidades regulatorias que han de permitir acercar

\footnotetext{
- El presente trabajo corresponde al tercer capítulo de la tesis a presentar con vistas a obtener el Doctorado en Economía, en el Departamento de Graduados de la Universidad Nacional del Sur.
} 
al óptimo el comportamiento del agente (el médico) impidiendo que el mismo intente manipular su nivel de esfuerzo en la atención de la salud de sus pacientes. El conflicto de intereses entre dichas partes surge principalmente por el tipo de contrato que posee el médico y los incentivos que le genera la función salarial sobre el nivel de esfuerzo con el que realiza la atención del agente. Diferentes contratos, es decir, diferentes funciones salariales pueden generar diferentes ineficiencias en el desempeño del médico lo cual requiere mecanismos correctores disímiles si se pretende minimizarlas. El desarrollo del tema se lleva a cabo en cuatro partes. En la primera se realiza un planteo teórico general que servirá de base para el desarrollo de los siguientes apartados. En la segunda parte se describe el funcionamiento de la atención médica en los hospitales públicos, la función de utilidad del médico (la cual responde al tipo de contrato laboral que posee) y el conflicto de intereses que este contrato genera en algunas variables de la función de utilidad del paciente. En la tercera parte se realiza el mismo análisis para el caso de los hospitales privados y en la cuarta parte se efectúa un análisis comparativo de las ineficiencias detectadas en el sector público en relación a las del sector privado, mostrando que, al responder cada médico a diferentes funciones de utilidad, su accionar, sus conflictos y las ineficiencias detectadas en relación a la atención del paciente resultan ser también diferentes según opere uno $u$ otro tipo de contrato salarial. Por último se describen las posibles herramientas regulatorias que, tanto en el sector público como en el privado, podrían reducir dichas ineficiencias.

\section{PARTE I \\ Marco teórico}

En el sector salud existen numerosas relaciones de agencia, cada una de las cuales reviste suma importancia debido a que de la interacción de las mismas resultan cuestiones que tienen que ver con la atención y el cuidado de la salud de los individuos. Entre las diversas relaciones se pueden citar: la relación político burócrata (donde el poder político delega en un burócrata la gestión de, por ejemplo, una obra social sindical), la relación del director de hospital con los médicos de la institución (donde el director le encomienda a los profesionales la tarea de atender a los pacientes que acuden a dicha institución), la relación médico - asociación intermedia (donde el médico delega en dicha asociación la facultad de administrar y negociar su tipo y monto de honorario con las obras sociales y medicinas prepagas, entre otras cosas). Sin embargo, una relación de agencia que cobra especial relevancia es aquella en la que interactúan el paciente como principal y el médico como agente, donde el paciente delega en el médico la tarea de atender su estado de salud. Es posible asumir que el médico tiene un mayor grado de información con respecto al paciente (existen asimetrías de información) donde el nivel de esfuerzo que realiza 
en dicha atención es una actitud muy difícil de verificar y monitorear (Macho Stadler, 1994). Este conflicto de intereses puede tener diferentes connotaciones según cuál sea la función salarial del profesional de la salud, es decir aquí entra en juego el mecanismo (o tipo de contrato) bajo el cual actúa el médico, asumiendo diferentes comportamientos según opere una u otra función salarial.

La función de utilidad de los médicos que desarrollan sus actividades dentro de un hospital está íntimamente vinculadas con:

i) La función objetivo de la institución hospitalaria, la cual puede estar orientada a la maximización del beneficio, la maximización del mercado o la maximización del bienestar social.

ii) El tipo de remuneración, que puede ser:

- salario fijo: implica una remuneración fija por una determinada cantidad de horas de trabajo y es independiente de la cantidad de pacientes atendidos. Esta forma de remuneración prevalece para casi todo el personal de los hospitales públicos, y en menor medida en el personal de los hospitales privados.

- salario por prestación: se remunera al profesional de acuerdo al número de prestaciones que ha realizado, es decir, su retribución es una función directa de la cantidad de atenciones llevadas a cabo. Este tipo de pago se da con más frecuencia en los médicos que pertenecen a los hospitales privados.

- salario por capitación: implica el pago de una remuneración fija por cada paciente atendido. Es decir, el profesional se compromete a la atención de un número determinado de pacientes por un monto determinado de dinero. Esta forma de pago prevalece en el caso de la atención de pacientes (generalmente en consultorios) con algún tipo de seguro de salud.

- pagos por tratamiento y por resultado: es posible encontrar también, esta alternativa como formas de pago dentro de un modelo organizacional, pero dado que estos tipos de pago son casi inexistentes en el sistema de salud argentino quedan excluidos en este tratamiento.

Tanto el objetivo de los hospitales como el tipo de remuneración pueden generar incentivos sustancialmente diferentes en el desempeño del profesional de la salud. Si bien no se pretende incorporar explícitamente la función objetivo hospitalaria en la función de utilidad de los profesionales de la salud, sí se incluirán otras variables que implícitamente responden al accionar institucional (salario, 
tiempo destinado a docencia, investigación y aprendizaje, entre otras). Se plantea una función de utilidad genérica del médico asumiendo que la misma está determinada por variables mensurables y no mensurables,

$$
\begin{gathered}
U_{\text {médico }}\left(\mathrm{x}_{\mathrm{i}}{ }^{\mathrm{m}}, \mathrm{y}_{\mathrm{i}}{ }^{\mathrm{m}}\right) \\
\text { dunde: } \\
\mathrm{x}_{\mathrm{i}}{ }^{\mathrm{m}} \text { variables mensurables del médico } \\
\mathrm{y}_{\mathrm{i}}{ }^{\mathrm{m}} \text { variables no mensurables del médico }
\end{gathered}
$$

Las $x_{i}^{m}$ representan las variables "salario" $y$ "horas trabajadas", las $y_{i}^{m}$ representan las variables "nivel de esfuerzo", "calidad", "especialización y aprendizaje". Cada una de estas variables genera utilidades o desutilidades en la función de preferencias del oferente de atención médica. Sin embargo, sólo algunas de estas variables se volverán operativas, según se trate de un médico en un hospital público o privado.

Desde el punto de vista de la demanda, los pacientes tienen diferentes funciones de utilidad de acuerdo a si demandan su atención en el sector público o en el privado, es decir, según internalicen o no los costos de su atención. Al igual que en el caso de los médicos se define una función de utilidad genérica para los pacientes en términos de variables mensurables y no mensurables,

$$
\begin{gathered}
\mathrm{U}_{\text {paciente }}\left(\mathrm{x}_{i}{ }^{\mathrm{p}}, \mathrm{y}^{\mathrm{p}}\right) \\
\text { donde, } \\
\mathrm{x}_{\mathrm{i}}{ }^{\mathrm{p}} \text { variables mensurables del paciente } \\
\mathrm{y}^{\mathrm{p}} \text { variable no mensurables del paciente }
\end{gathered}
$$

Las $x_{i}^{p}$ representan las variables "precio pagado", "cantidad de prestaciones demandadas por el médico" y "tiempo de espera". La única variable intangible $y^{p}$ es "la calidad de la atención recibida". Cada una de estas variables genera utilidades o desutilidades sobre la función de preferencias del paciente.

Se asume que la sumatoria de las funciones de utilidad de los médicos está representada por una función $\psi$ :

$$
\Psi\left(x^{m}, y^{m}\right)=\sum_{l=1}^{m} U j\left(x_{i}{ }^{m}, y_{i}^{m}\right) \quad \text { con } j=1, \ldots \ldots, n
$$


y que la sumatoria de las funciones de utilidad de los pacientes esta representada por la función $\delta$ :

$$
\delta\left(\mathrm{x}^{\mathrm{p}}, \mathrm{y}^{\mathrm{p}}\right)=\sum_{\mathrm{l}=1}^{\mathrm{m}} \mathrm{U}_{1}\left(\mathrm{x}_{\mathrm{i}}^{\mathrm{p}}, \mathrm{y}^{\mathrm{p}}\right) \quad \text { con } \mathrm{l}=1, \ldots \ldots, \mathrm{m}
$$

En equilibrio deberian verificarse simultáneamente las siguientes condiciones:

$$
\begin{array}{cc}
\text { Sujeto a } & \begin{array}{c}
\operatorname{Max} \Psi\left(\mathrm{x}^{\mathrm{m}}, \mathrm{y}^{\mathrm{m}}\right) \\
\delta\left(\mathrm{x}^{\mathrm{p}}, \mathrm{y}^{\mathrm{p}}\right) \\
\mathrm{y}
\end{array} \\
\text { Sujeto a } & \left.\begin{array}{c}
\operatorname{Max} \delta\left(\mathrm{x}^{\mathrm{p}}, \mathrm{y}^{\mathrm{p}}\right) \\
\Psi
\end{array} \mathrm{x}^{\mathrm{m}}, \mathrm{y}^{\mathrm{m}}\right) \quad \text { dada }
\end{array}
$$

dándose una situación de equilibrio de Nash. La combinación de variables tangibles y no tangibles comunes a médicos y pacientes deben, en equilibrio, alcanzar los mismos valores.

La principal razón que puede ocasionar un desequilibrio es la repercusión de la asimetría de información que existe entre el médico y el paciente. El médico es contratado por el paciente para ofrecerle un diagnóstico sobre su estado de salud o un tratamiento en caso que necesite un procedimiento para mejorarlo. La dificultad que enfrenta el paciente para determinar si lo prescripto por el médico es necesario y conveniente impide que, en general, se llegue a una situación eficiente. A este tipo de bienes donde es dificil medir la calidad del servicio ofrecido se los denomina bienes de confianza (credence goods). En los bienes de confianza es necesario delegar la valoración de la calidad a quienes proveen el servicio. Esto hace necesario un mecanismo que discipline a los expertos y les dé incentivos para perseguir los objetivos deseados: contención de costos y calidad. El problema de incentivos en los médicos está ligado a su poder de decisión sobre la calidad del diagnóstico, su poder de manipulación sobre la percepción del estado de salud del enfermo o sus posibles consecuencias y la posibilidad de inducir al paciente a demandar más o menos tratamiento. Está, por lo tanto, relacionado a su poder de explotar la existencia de asimetrías informativas. La evidencia empírica parece sugerir que los médicos abusan de su rol de agente del paciente actuando en su propio interés. No obstante, este aprovechamiento de las asimetrías de información está condicionado al sistema de pago de los servicios. Por esta razón es conveniente analizar los incentivos que genera cada función salarial y sus efectos sobre la función de utilidad de los pacientes. 
A continuación se introduce el accionar del director de hospital, quien debe diseñar los contratos salariales adecuados. Este nuevo actor tiene especial relevancia debido a que los contratos que rigen en su institución tendrán consecuencias inmediatas sobre la atención recibida por los pacientes. Si el director de cada hospital, público o privado, se propone internalizar las funciones de preferencias de los médicos y los pacientes, además de optimizar los recursos monetarios, físicos y humanos, deberá considerar el sistema institucional que junto con las restricciones presupuestarias defina los niveles de utilidad del médico y del paciente en el equilibrio. Por lo tanto, el resultado de este sistema institucional (o mecanismo) se deberá evaluar a través de las variables tangibles e intangibles que identifican las respectivas funciones de preferencias.

La idea que subyace al planteo anterior es que el Director del hospital o planificador debería encontrar un mecanismo $\mathbf{M}$ a través del cual logre alcanzar, con los recursos monetarios ( $\mathrm{rm}$ ), físicos (rf) y humanos ( $\mathrm{rh}$ ) disponibles, un nivel de la función de preferencia social que involucre la mejor combinación de las variables $x$ e y que satisfaga la condición de equilibrio entre las funciones de utilidad de los médicos y los pacientes.

La combinación de variables $\psi$ y $\delta$ de las funciones debería surgir de la función de preferencia social $\varphi$ definida por el director del hospital e implementada a través del mecanismo $\mathbf{M}$.

$$
\begin{gathered}
\mathrm{M}\{\mathrm{rm}, \mathrm{rf}, \mathrm{rh}\} \rightarrow\left\{\mathrm{x}_{\mathrm{i}}^{\mathrm{m}}, \mathrm{y}_{\mathrm{i}}^{\mathrm{m}}, \mathrm{x}^{\mathrm{p}}, \mathrm{x}^{\mathrm{p}}\right\} \\
\text { tal que } \\
\underset{\mathrm{rm}, \mathrm{rf}, \mathrm{rh}, \mathrm{x}^{\mathrm{m}}, y^{m}, x}{\mathrm{Max}_{, y} p} \quad \phi\left(\mathrm{rm}, \mathrm{rf}, \mathrm{rh}, \mathrm{x}_{\mathrm{i}}{ }^{\mathrm{m}}, \mathrm{y}_{\mathrm{i}}^{\mathrm{m}}, \mathrm{x}_{\mathrm{i}}^{\mathrm{p}} \mathrm{y}^{\mathrm{p}}\right)
\end{gathered}
$$

La figura del director que implementa la función de preferencia social constituye una instancia de un planificador omnisciente y benevolente que busca el interés de los médicos y los pacientes conociendo sus deseos y preferencias. Sin embargo, este planteo se enfrenta con dos dificultades principales. La primera es que resulta poco realista suponer que el director se comporta sólo en función de los intereses de los médicos y los pacientes (en vez de buscar la consecución de sus intereses personales) y la segunda es exigir demasiado que pueda estimar exactamente sus utilidades.

Dado el rol decisivo de los profesionales de la salud en el funcionamiento 
de la institución hospitalaria, para que el planificador encuentre un estado de equilibrio se requerirá que inđuzca las decisiones de los médicos a través de un mecanismo de incentivos (conjunto de reglas o tipo de contrato) $\mathbf{M}$ que logre acercar las variables compartidas entre los médicos y los pacientes.

El mecanismo $\mathbf{M}$ de asignación de recursos monetarios, físicos y humanos que determina un estado de equilibrio (en relación a las,variables tangibles y no tangibles) deberá inducir decisiones individuales racionales y eficientes en el sentido de Pareto. Esto indica que el planificador no podrá implementar un mecanismo $\mathbf{M}^{\prime}$ de asignación de recursos que implique una mejora para los médicos sin que esto incluya una pérdida para los pacientes, o viceversa. Se puede afirmar entonces que, la aplicación de un mecanismo $\mathbf{M}$ determina una asignación de recursos tal que no existe otro mecanismo $\mathbf{M}^{\prime}$ por el cual se obtenga una asignación de recursos preferida a la anterior, es decir el mecanismo $\mathbf{M}$ determina un estado Pareto eficiente si:

$$
\begin{aligned}
& E^{M}\left(x^{m}, y^{m}\right) \geq E^{M}\left(x^{m}, y^{m}\right) \\
& E^{M}\left(x^{p}, y^{p}\right) \geq E^{M^{\prime}}\left(x^{p}, y^{p}\right)
\end{aligned}
$$

Es decir, el estado al que arriban los médicos y los pacientes (en términos de las variables tangibles y no tangibles de sus respectivas funciones de utilidad) con el mecanismo de asignación de recursos (monetarios, fisicos y humanos) $\mathbf{M}$ adoptado por el director del hospital es superior en términos de Pareto al estado que le determinaría cualquier otro mecanismo $\mathbf{M}^{\prime}$.

Un conjunto de reglas diseñado para que los médicos y los pacientes coordinen sus acciones debe basarse en un sistema de información eficiente. Sin embargo, uno de los problemas centrales del funcionamiento de las instituciones hospitalarias es la obtención, el procesamiento y la comunicación de la información, por lo que puede resultar poco factible la transmisión y el procesamiento de todos los datos que el planificador necesita para resolver el problema de la optimización en la asignación de recursos.

Lo planteado en los párrafos anteriores puede ser analizado como una aplicación del concepto de "optimalidad" a un proceso de asignación de recursos y diseño de contratos (Corchón, 1996). A la luz de este enfoque se analizan los diferentes contratos laborales que rigen el accionar de los médicos en las instituciones hospitalarias y los incentivos que éstos generan sobre la atención de los pacientes. Si bien en los análisis posteriores no aparecerá en forma explícita la figura del director del hospital, la misma estará presente debido a que es quien diseña los 
contratos laborales.

Los diferentes tipos de contratos que operan en la relación médico paciente según el contexto sea un hospital público o privado se caracterizan por definir funciones salariales diferentes. Esto genera diferentes conflictos, diferentes ineficiencias y diferentes necesidades regulatorias si se desea diseñar un contrato que acerque los valores óptimos de las variables compartidas tanto a los niveles deseados del médico como del paciente.

PARTE II

La atención médica en los hospitales públicos

Los servicios ofrecidos por los hospitales públicos son diversos, poseyendo cada tipo de servicio una estructura de mercado diferente y si bien coexisten en una misma unidad funcional responden a diferentes patrones de comportamiento. A continuación se describe el funcionamiento del servicio denominado genéricamente "atención médica".

\section{a) Características del servicio}

El servicio brindado por los profesionales médicos dentro de los hospitales públicos tiene la característica de "un bien privado suministrado públicamente", esto es, satisface la condición de consumo rival pero no es posible aplicar el principio de exclusión. La provisión de este servicio genera beneficios y costes externos. Los beneficios no sólo se internalizan en cada uno de los individuos que reciben atención médica sino que se extienden a toda la comunidad que se ve beneficiada por el mejor nivel de salud y el menor riesgo de enfermar o morir de los individuos. Los beneficios externos tienen su contrapartida en los costos externos que se derivan del proceso de financiamiento del servicio. Sin embargo el coste de este servicio dificilmente sea internalizado por los consumidores directos del mismo. En general tal provisión está financiada a través de un presupuesto cuyo origen es impositivo y está asignado por el gobierno.

b) Oferta y demanda

El servicio de atención médica es ofrecido por los profesionales de la salud quienes atienden las consultas de los pacientes en el ámbito de los hospitales. El médico es el primer agente al que recurren las personas cuando creen tener un problema de salud que no pueden resolver por su cuenta, por lo tanto es quien decide sobre el diagnóstico, tratamiento, prescripción de medicamentos y que deriva 
a los pacientes a otros servicios médicos. Este servicio tiene un rol muy importante dentro del funcionamiento hospitalario ya que de él depende gran parte de las demandas a los demás servicios. Es decir, la atención médica es responsable de demandar o no más servicios dentro del mismo hospital. Es por eso que la atención brindada por un médico juega un rol clave en el proceso de producción y distribución de todo el resto de los servicios médicos.

El hecho clave en la relación médico-paciente es que el demandante (paciente) no tiene información suficiente acerca de sus verdaderas necesidades. Un paciente que consulta al médico sólo sabe que requiere tratamiento médico porque no está satisfecho con su estado de salud. Pero delega la elección del tratamiento al médico, a quien considera mejor informado y luego decidirá si actúa o no de acuerdo a lo que éste prescribe. Por lo tanto, esto significa que la curva de demanda, que describe el volumen de uso de servicios médicos refleja predominantemente las decisiones de los oferentes de cuidados médicos y no de los pacientes. En el límite, se puede decir que la demanda de cuidados médicos está determinada por el oferente (Lavila,1999).

El argumento anterior plantea la hipótesis de que la demanda de servicios médicos es inducida por parte de los profesionales de la salud. En el caso de los hospitales públicos esta conducta de los médicos no parece tener un objetivo económico, sino más bien es el comportamiento habitual del accionar médico. Es decir, esa conducta de los oferentes como generadores de demanda no explica más que su rol dentro del establecimiento hospitalario, y no implica la posibilidad de un comportamiento maximizador de beneficios dado que en estos hospitales opera una restricción salarial y el ingreso no es función de las prestaciones realizadas.

Los profesionales que brindan este servicio en los hospitales públicos generalmente destinan parte de su tiempo a actividades de docencia, especialización y aprendizaje dentro del mismo hospital, razón por la cual ven atractiva su función en el sector público. La posibilidad de incrementar su nivel de conocimiento compensa la desutilidad que les implica la atención de un gran número de pacientes por un mismo salario. A diferencia del sector privado en el que, en muchos casos, la remuneración es proporcional a los pacientes atendidos, en el sector público perciben un salario por una determinada cantidad de horas trabajadas independientemente del número de pacientes atendidos.

Si bien la oferta de los profesionales de la salud en los hospitales públicos abarca la mayoría de las especialidades médicas, en los últimos años en Argentina su capacidad como oferente de servicios ha sido ampliamente superada por un exceso de demanda que no encuentra contención en los servicios de salud del sector 
privado. La imposibilidad de excluir a los individuos del consumo del servicio genera la necesidad de controlar ese exceso de demanda a través de diferentes formas, siendo la más habitual la utilización de listas de espera.

c) Funciones de utilidad

De acuerdo a lo planteado anteriormente la cantidad ofrecida de bienes y servicios para el cuidado de la salud no necesariamente iguala a la cantidad que los pacientes demandarían si estuviesen perfectamente informados. Esta asimetría de información entre el médico y el paciente puede modelarse en términos de la relación Agente-Principal. A continuación se describen las funciones de utilidad de cada uno de ellos y el conflicto de intereses que se genera sobre el nivel de esfuerzo del médico debido al tipo de contrato laboral que posea con la institución hospitalaria.

\section{El médico como agente}

La función de utilidad del oferente de servicios médicos se puede analizar a partir de las cuatro variables que determinan su preferencia por pertenecer a un hospital público:

$$
U_{\text {médico }}: U_{\text {médico }}(w, h s, e \& a, e)
$$

Donde la variable w representa el salario del médico. Dicho salario es un monto fijo ya que no cobra por el número de prestaciones que realiza y para simplificar suponemos que no existe el pago por horas extras, con lo cual podemos inferir que su salario esta determinado exógenamente. La función de utilidad tiene, con respecto al salario, una relación positiva decreciente:

$$
\frac{\delta U_{\text {médico }}}{\delta w}>0, \frac{\delta^{2} U_{\text {médico }}}{\delta w^{2}} \leq 0
$$

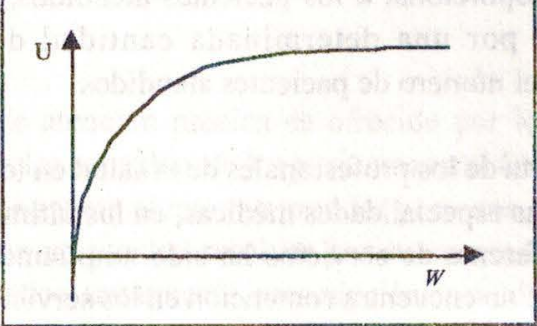


La variable hs. $(0 \leq \mathrm{hs} . \leq 24)$ representa las horas trabajadas del médico por día en el hospital público. Si bien se trata normalmente de una jornada laboral de ocho horas, pueden existir acuerdos laborales que impliquen las 24 horas, como en el caso de los profesionales que sólo realizan guardias. La relación con la función de utilidad es continuamente decreciente, es decir:

$$
\frac{\delta U_{\text {médico }}}{\delta h s}<0, \frac{\delta^{2} U_{\text {médico }}}{\delta h s^{2}}<0
$$

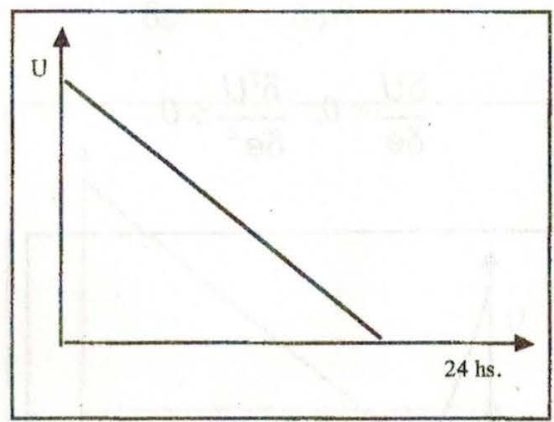

La variable e \& $\mathbf{a}^{1}$ incorpora las actividades de docencia, especialización y aprendizaje que realiza el médico que pertenece a una institución hospitalaria pública. La relación con la función de utilidad es creciente, pero a una tasa decreciente, es decir:

$$
\frac{\delta U_{\text {médico }}}{\delta(\mathrm{e} \& \mathrm{a})}>0, \frac{\delta^{2} U_{\text {médico }}}{\delta(\mathrm{e} \& \mathrm{a})^{2}} \leq 0
$$

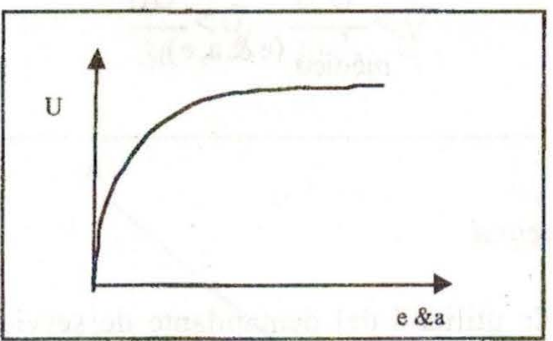

' La variable e\&a denota las actividades que realiza el médico a diferencia de la variable e que únicamente refleja el nivel de esfuerzo. 
La variable e indica el nivel de esfuerzo que debe realizar el médico dentro del hospital público para atender a sus pacientes. La relación de dependencia que tiene el profesional de la salud con la institución hospitalaria lo obliga a permanecer en ella una cantidad determinada de horas. El número de pacientes que debe atender no es una variable de control para el médico, normalmente es un número suficientemente alto (debido a la gran congestión) por lo que el nivel de esfuerzo del profesional por realizar dicha atención es una variable relevante en su función de utilidad. Dado que el nivel de esfuerzo es independiente del salario percibido, la relación que tiene dicho esfuerzo con la función de utilidad es continuamente decreciente, esto es:

$$
\frac{\delta U}{\delta e}<0, \frac{\delta^{2} U}{\delta e^{2}} \leq 0
$$

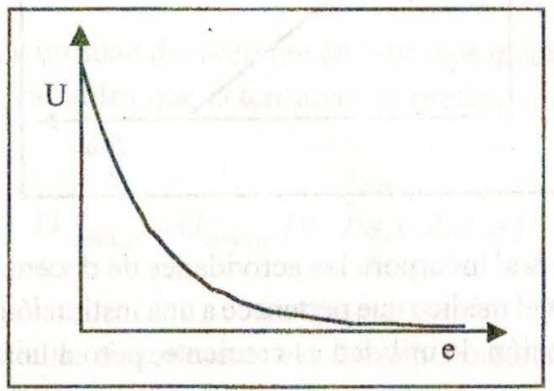

Dado que en el hospital público tanto el salario (w) como las horas trabajadas (hs) son variables determinadas exógenamente para el médico, su función de utilidad puede ser reducida a:

$$
U_{\text {médico }}(\mathrm{e} \& \mathrm{a}, \mathrm{e})
$$

El paciente como principal

La función de utilidad del demandante de servicios médicos en los hospitales públicos se determina a partir de las tres variables que se detallan a continuación: 


$$
\mathrm{U}_{\text {paciente }}: \mathrm{U}_{\text {paciente }}(\mathrm{p}, \mathrm{t}, \mathrm{c})
$$

Donde p representa el precio que el paciente debe pagar por la atención que el médico le dispense. La función de utilidad del paciente con respecto al precio que debe pagar por su atención es estrictamente decrecieṇte.

$$
\frac{\delta U}{\delta p}<0, \quad \frac{\delta^{2} U}{\delta 0^{2}}<0
$$

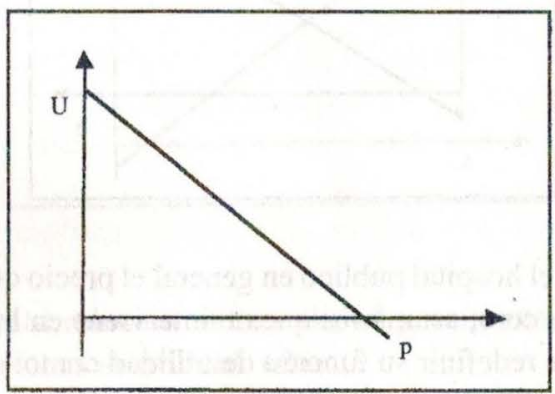

La variable $\mathbf{t}$ representa el tiempo de espera en que el paciente incurre para ser atendido por el médico del hospital público. Al igual que el precio, el tiempo de espera también influye negativamente en la función de utilidad del paciente, es decir, la función de utilidad con respecto a esta variable es estrictamente decreciente.

$$
\frac{\delta U}{\delta t}<0, \quad \frac{\delta^{2} U}{\delta !^{2}}<0
$$

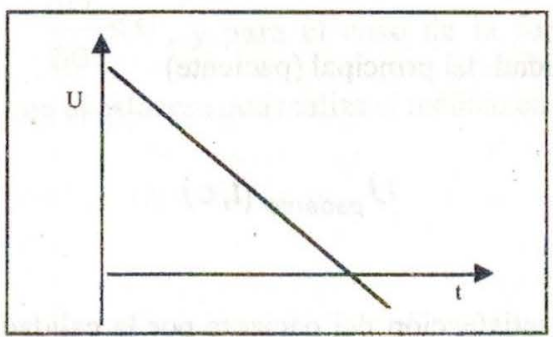


La variable ces la calidad de la atención recibida de parte del médico. La función de utilidad del paciente tiene una relación estrictamente positiva con respecto a esta variable, esto es:

$$
\frac{\delta U}{\delta c}>0, \frac{\delta^{2} U}{\delta c^{2}}>0
$$

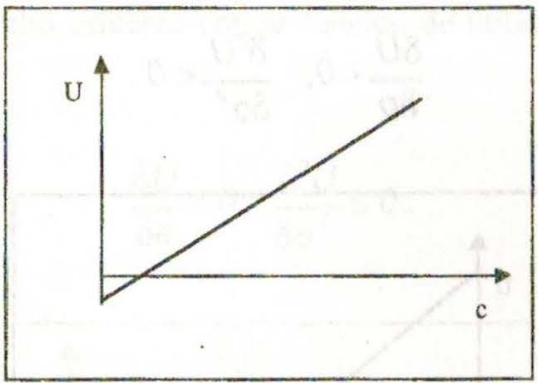

Dado que en el hospital público en general el precio que deben abonar por la atención recibida es cero, asumimos que no interviene en la función de utilidad del paciente. Se puede redefinir su función de utilidad como:

$$
U_{\text {paciente }}(t, c)
$$

Conflicto de intereses entre el agente y el principal

A partir de la función de utilidad del agente (médico):

$$
U_{\text {médico }}(e \& a, e)
$$

y de la función de utilidad del principal (paciente):

$$
U_{\text {paciente }}(t, c)
$$

Dado que la satisfacción del paciente por la calidad de la atención que 
recibe está en directa relación con el nivel de esfuerzo que realice el médico en el cuidado de su salud, se define la calidad de la atención médica (c) como una función directamente proporcional al esfuerzo realizado por el médico, es decir:

$$
\begin{aligned}
& c=c(e) \quad \text { donde: } \\
& c^{\prime}(e)>0, \quad c^{\prime \prime}(e)>0
\end{aligned}
$$

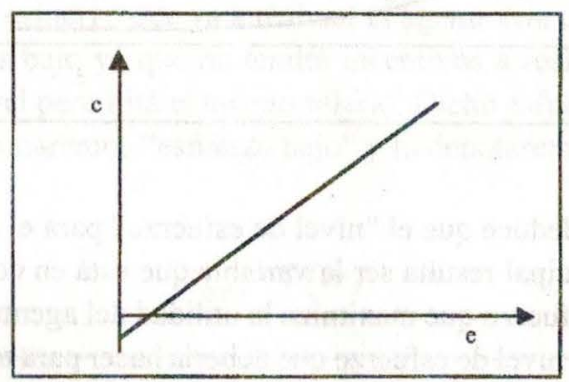

Definida esta relación entre calidad y esfuerzo, se pueden redefinir nuevamente las funciones de utilidad como:

$$
U_{\text {médico }}(e \& a, e) \text { para el agente }
$$

$U_{\text {paciente }}(t, e) \quad$ para el principal

El esfuerzo es una variable común en ambas funciones de utilidad. Sin embargo en cada una de ellas la relación con el nivel de esfuerzo es distinta. En el caso de la función de utilidad del médico la relación con el esfuerzo que realiza es negativa, es decir, $\frac{\delta U}{\delta e}<0$, y para el caso de la función de utilidad del paciente la relación con el esfuerzo que realiza el médico en la atención es positiva, esto es, $\frac{\delta U}{\delta e}>0$.

Gráficamente: 


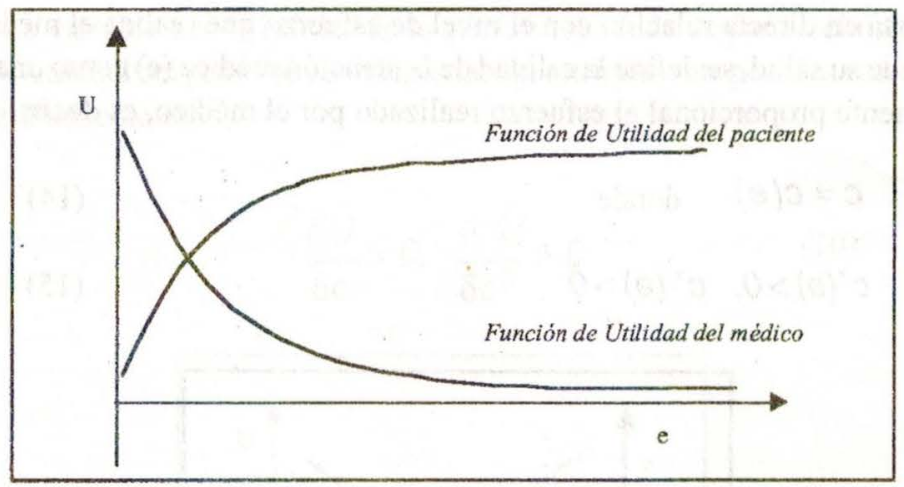

De esto se deduce que el "nivel de esfuerzo" para el agente o "la calidad recibida" por el principal resulta ser la variable que está en conflicto entre dichas partes. El nivel de esfuerzo que maximiza la utilidad del agente no necesariamente debe coincidir con el nivel de esfuerzo que debería hacer para maximizar la utilidad del principal ya que lo que para el paciente representa utilidad resulta una desutilidad para el médico.

Este problema implicaría en otros casos de relación de agencia la necesidad de que el principal formule un contrato que acerque al óptimo el comportamiento del agente. Sin embargo en este caso el principal (paciente) no es quien determina las reglas para controlar el comportamiento del agente (médico). Lo que determina que el médico quiera minimizar su nivel de esfuerzo en este caso es la forma de pago o el contrato laboral diseñado por el director de la institución hospitalaria que en el caso de los hospitales públicos estipula un salario fijo por una determinada cantidad de horas.

En este contexto es posible definir el tipo de esfuerzo en términos de la función salarial (w) del médico, es decir, se redefine el esfuerzo como una función del salario percibido:

$$
e=f(w)
$$

A su vez la función salarial se la puede expresar como:

$$
w=f(P)=\vec{w}+k P \quad \text { dónde } P \text { es el número de prestaciones (19) }
$$

El salario del agente se definiría entonces como una función lineal en la que el primer término es una constante que indica el salario fijo, y el segundo 
término es la parte del salario que se percibe en el caso que pueda recibir ingresos por el número de prestaciones que realice. En el caso del hospital público el número de prestaciones $P$ no es una variable de control para el médico por lo tanto la función del salario se reduce sólo al primer término:

$$
w=\bar{w}
$$

El nivel de esfuerzo que va a realizar el agente con una remuneración de este tipo será el más bajo ya que no tendrá incentivos a realizar un nivel alto si cualquiera sea el nivel percibirá el mismo salario. Dicho esfuerzo será entonces el menor posible, lo llamaremos "esfuerzo bajo" y lo denotaremos e bajo $_{\text {, }}$ es decir:

$$
e_{b a j o}=f(\bar{w})
$$

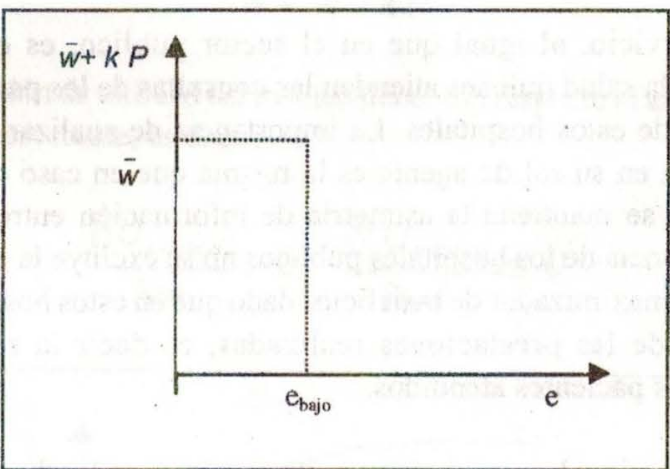

\section{PARTE III}

La atención médica en los hospitales privados

Los servicios ofrecidos por los hospitales privados al igual que en los hospitales públicos son diversos. Cada uno posee una estructura de mercado diferente y si bien coexisten en una misma unidad funcional responden a diferentes patrones de comportamiento. A continuación se describe el funcionamiento del servicio denominado "atención médica".

a) Características del servicio 
El servicio brindado por los profesionales médicos dentro de los hospitales privados tiene la característica de "un bien privado", ya que satisface la condición de consumo rival y además es posible aplicar el principio de exclusión. El servicio ofrecido por los profesionales de la salud es brindado únicamente contra una contrapartida económica, ya sea con dinero circulante o con algún tipo de bono suministrado por una compañía de seguros de salud. Además de las características mencionadas, la provisión de este servicio genera beneficios y costes externos. Los beneficios, al igual que en el sector público, no sólo se internalizan en cada uno de los individuos que reciben atención médica sino en toda la comunidad que se ve beneficiada por el mayor stock de salud y el menor riesgo de enfermar o morir. El fenómeno de los beneficios externos tiene su contrapartida en los costes externos que provienen del aporte realizado por el paciente. De esto se deduce que, a diferencia del sector público, el costo del servicio es internalizado por el consumidor del mismo.

b) Oferta y demanda

Este servicio, al igual que en el sector público, es ofrecido por los profesionales de la salud quienes atienden las consultas de los pacientes dentro de los consultorios de estos hospitales. La importancia de analizar la conducta que adopte el médico en su rol de agente es la misma que en caso de los hospitales públicos, ya que se mantiene la asimetría de información entre el médico y el paciente. A diferencia de los hospitales públicos no se excluye la posibilidad de un comportamiento maximizador de beneficios dado que en estos hospitales el ingreso es una función de las prestaciones realizadas, es decir la remuneración es proporcional a los pacientes atendidos.

Los profesionales que brindan este servicio en los hospitales privados generalmente no destinan tiempo a actividades de especialización y aprendizaje dentro de dicho hospital, razón por la cual en muchos casos el mismo profesional que está en un hospital privado también presta servicios en uno del sector público donde sí ejerce dichas actividades.

La demanda de este servicio la realizan únicamente pacientes con fondos suficientes como para afrontar en forma particular sus gastos en salud, o pacientes con algún tipo de cobertura sanitaria (obra social o medicina prepaga), es decir, con algún tipo de seguro de salud.

c) Funciones de utilidad

También la asimetría de información entre el médico y el paciente existe 
en el sector privado, por esta razón es posible nuevamente modelar dicha situación en términos de la relación agente-principal. A continuación se describen las funciones de utilidad de cada una de las partes y el conflicto de intereses que resulta de su interacción.

\section{El médico como agente}

La función de utilidad del oferente de servicios médicos se formula a partir de tres variables que predominantemente determinan su preferencia por pertenecer a un servicio del sector privado y viene dada por:

$$
U_{\text {médico }}: U_{\text {médico }}(w, h s ., e)
$$

Donde la variable w representa el salario del médico. Este salario es una función directamente proporcional al número de prestaciones que realiza, es decir:

$$
w=\bar{w}+k P
$$

La función de utilidad del médico tiene, con respecto al salario, una relación positiva pero decreciente, es decir:

$$
\frac{\delta U_{\text {médico }}}{\delta w}>0, \frac{\delta^{2} U_{\text {médico }}}{\delta w^{2}} \leq 0
$$

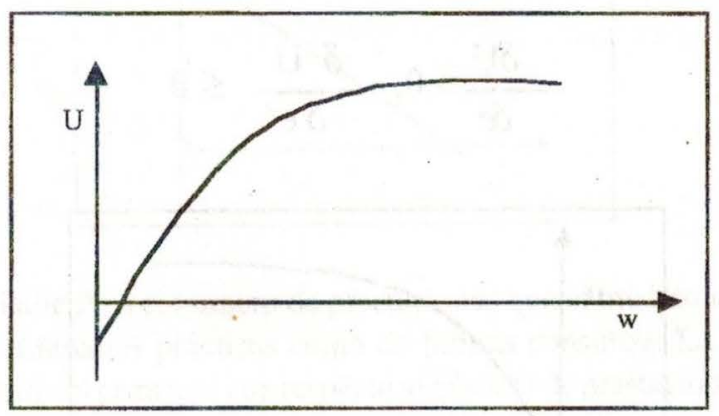

La variable hs. representa las horas trabajadas del médico por día en el hospital privado. Esta es una variable de control para el médico ya que él decide qué cantidad de prestaciones realizar y por lo tanto el tiempo que le insume será proporcional al número de pacientes atendidos. La función de utilidad es continuamente decreciente en el número de horas trabajadas, es decir: 


$$
\frac{\delta \mathrm{U}}{\delta \mathrm{hs}}<0, \quad \frac{\delta^{2} \mathrm{U}}{\delta \mathrm{hs}^{2}}<0
$$

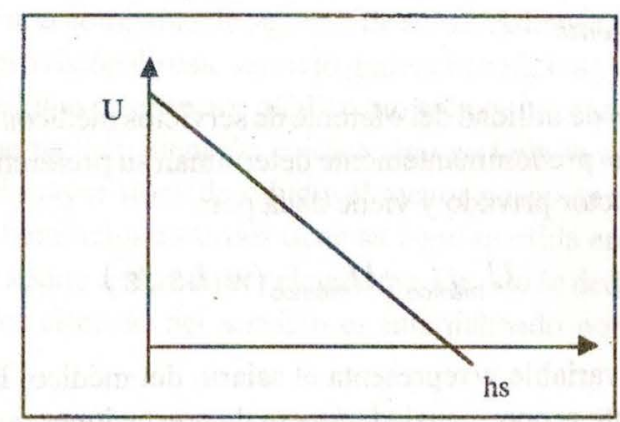

La variable e representa el nivel de esfuerzo realizado por el profesional de la salud en la atención de sus pacientes. Pero en este caso, a diferencia del hospital público, la relación con la función de utilidad será creciente. La razón de esta diferencia se encuentra en que del esfuerzo que realice en la atención de sus pacientes dependerán las posteriores consultas de los mismos y por lo tanto su ingreso presente y futuro. Dado que el nivel de esfuerzo determina el salario percibido, la relación que tiene dicho esfuerzo con la función de utilidad es creciente, pero a una tasa decreciente, es decir:

$$
\frac{\delta \mathrm{U}}{\delta e}>0, \quad \frac{\delta^{2} \mathrm{U}}{\delta \mathrm{e}^{2}} \leq 0
$$

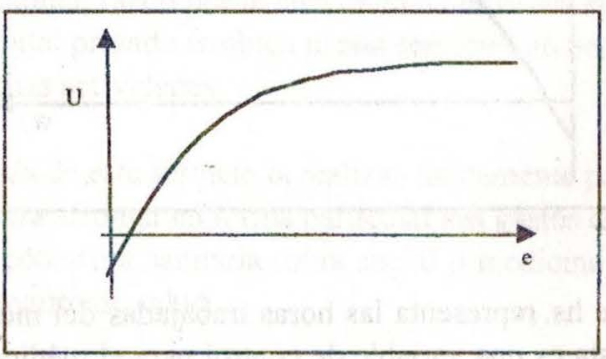

Una vez definidas las variables que intervienen en la función de utilidad del oferente de servicios médicos se puede redefinir la función como: 


$$
U_{\text {medico }}(w, h s, e)
$$

El paciente como principal

La función de utilidad del demandante de servicios médicos, es decir del principal, en los hospitales privados se determina a partir de las tres variables que se detallan a continuación:

$$
U_{\text {paciente }}=U_{\text {paciente }}(t, P, c)
$$

Donde trepresenta el tiempo de espera para ser atendido por el médico del hospital privado. El tiempo de espera influye negativamente en la función de utilidad del paciente, es decir, la función de utilidad con respecto a esta variable es estrictamente decreciente.

$$
\frac{\delta U}{\delta t}<0, \quad \frac{\delta^{2} U}{\delta t^{2}}<0
$$

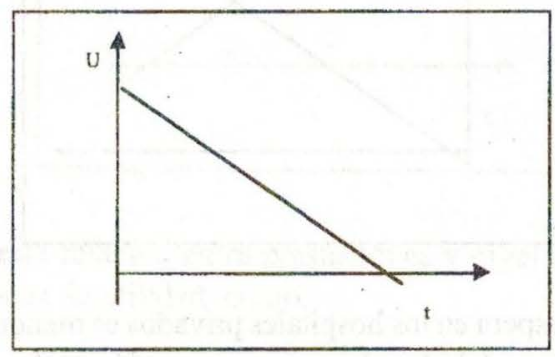

La variable $\mathbf{P}$ es el número de prestaciones que el médico le demanda, ya sea a través de diferentes prácticas como de futuras consultas. La relación de la función de utilidad del principal con respecto al número de prestaciones es negativa, es decir:

$$
\frac{\delta U}{\delta P}<0, \quad \frac{\delta^{2} U}{\delta P^{2}} \leq 0
$$

Esto último descansa en el argumento de que cada prestación demandada por el médico le implica al paciente un desembolso monetario y una determinada 
cantidad de tiempo.

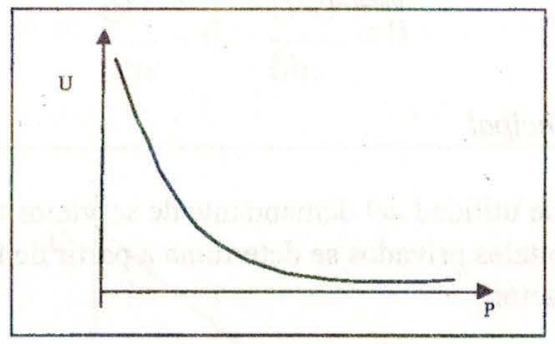

La variable c explica cómo actúa en la función de utilidad del paciente la calidad de la atención recibida por el médico. La función de utilidad del paciente tiene una relación estrictamente positiva con respecto a esta variable, esto es:

$$
\frac{\delta U}{\delta c}>0, \quad \frac{\delta^{2} U}{\delta c^{2}}>0
$$

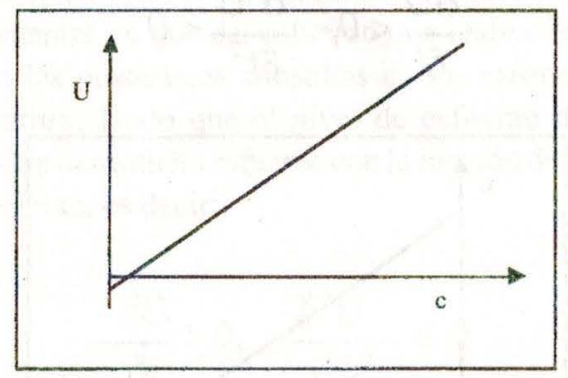

El tiempo de espera en los hospitales privados es menor al tiempo de espera en los hospitales públicos debido a la menor congestión y el más fácil acceso. Por esta razón dicha variable no se vuelve relevante en el análisis. Se supone que es igual a cero, y que no interviene en la función de utilidad del principal. Se puede redefinir la función de utilidad como:

$$
U_{\text {paciente }}(P, c)
$$

Conflicto de intereses entre el agente y el principal

A partir de la función de utilidad del médico: 


$$
U_{\text {médico }}(w, h s, e)
$$

y de la función de utilidad del paciente:

$$
U_{\text {paciente }}(P, C)
$$

Es posible definir el número de prestaciones como una función directamente proporcional al esfuerzo del médico ya que del esfuerzo que realice por generar diversas prácticas y posteriores consultas dependerá tanto su ingreso presente como su ingreso futuro, esto es:

$$
\begin{aligned}
& P=P(e) \quad \text { dónde: } \\
& P^{\prime}(e)>0, \quad P^{\prime \prime}(e)>0
\end{aligned}
$$

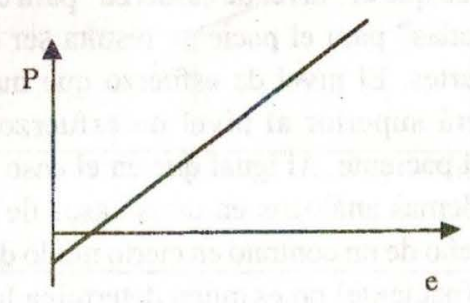

Definida esta relación entre prestaciones y nivel de esfuerzo, se pueden redefinir las funciones de utilidad como:

$$
\begin{gathered}
U_{\text {médico }}(W, h s, e) \\
y \\
U_{\text {paciente }}(e, c)
\end{gathered}
$$

En cada una de estas funciones de utilidad el nivel de esfuerzo influye en forma distinta. En el caso de la función de utilidad del médico la relación con el esfuerzo que realiza es positiva y para el caso de la función de utilidad del paciente la relación con el esfuerzo que realiza el médico en la atención es negativa. 
Gráficamente:

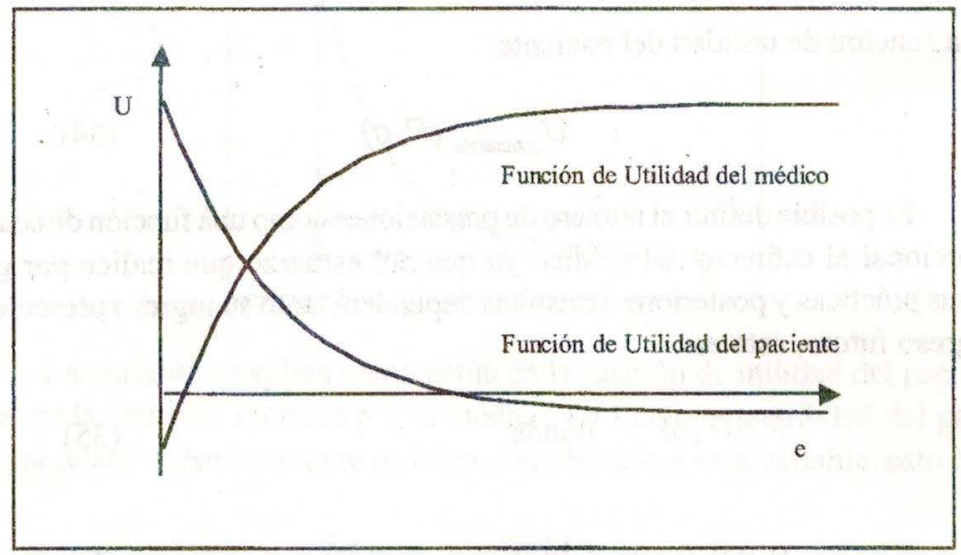

De aquí se deduce que el "nivel de esfuerzo" para el médico o "la cantidad de prestaciones demandadas" para el paciente resulta ser la variable que está en conflicto entre dichas partes. El nivel de esfuerzo que maximiza la utilidad del médico seguramente será superior al nivel de esfuerzo que debe hacer para maximizar la utilidad del paciente. Al igual que en el caso del hospital público se plantea que si bien problemas análogos en otros casos de relación de agencia se resuelven mediante el diseño de un contrato en cierto modo definido por el principal, en este caso el principal (paciente) no es quien determina las reglas para controlar el comportamiento del agente (médico). Lo que determina que el agente quiera maximizar su nivel de esfuerzo es nuevamente la función salarial que le otorgó el director de la institución hospitalaria, es decir, el cobro del salario en función del número de prestaciones. Por lo tanto, se define el conflicto de intereses en relación al salario (w) y el nivel se esfuerzo (e). El esfuerzo es una función del tipo de contrato salarial:

$$
\begin{gathered}
e=f(w) \\
w=\bar{w}+k P
\end{gathered}
$$

Dado que el salario del agente en los hospitales privados está determinado generalmente por un salario fijo más una parte variable que depende del número de prestaciones que lleve a cabo, dichas prestaciones constituyen una variable de control para el médico. El nivel de esfuerzo que va a realizar el agente con una remuneración de este tipo será el más alto posible dada una restricción de tipo ética, que suponemos 
es $\mathrm{P}=\mathrm{P}^{*}$ donde más allá de dicho número de prestaciones se verá desprestigiado por una potencial sobre-prestación. Respetando ese máximo de prestaciones dado por $\mathrm{P} *$ tendrá incentivos a generar el mayor número posible de prácticas médicas y garantizarse un cierto nivel de ingresos. Es posible decir que el nivel de esfuerzo será entonces el mayor posible, lo llamaremos "esfuerzo alto" y lo denotaremos $\operatorname{con} \mathrm{e}_{\text {alto }}$, es decir:

$$
e_{\text {alto }}=f\left(\bar{w}+k P^{*}\right)
$$

Gráficamente:

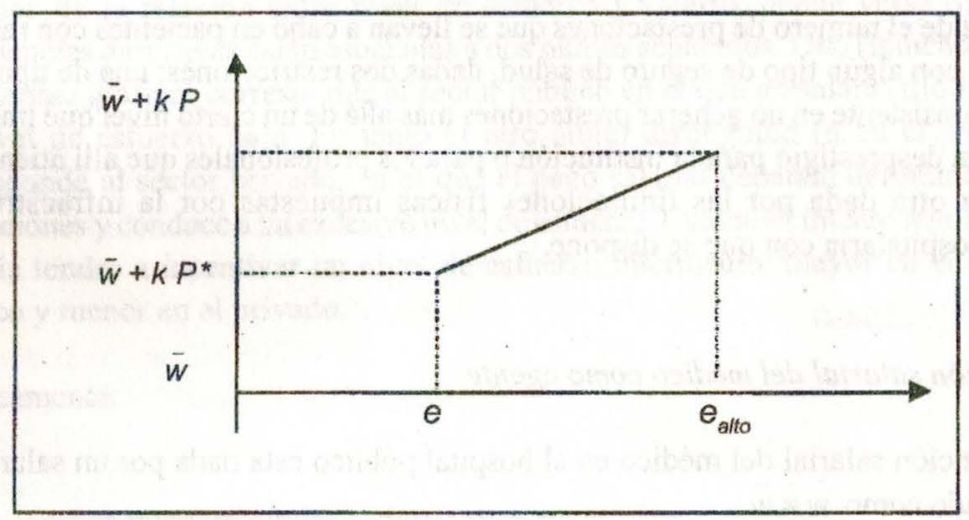

PARTE IV

Análisis comparativo

En las partes II y III se analizó la relación de agencia entre el médico y el paciente bajo el conjunto de reglas que rigen respectivamente al hospital público y al hospital privado. En cada apartado esta relación de agencia derivó en diferentes conflictos de intereses. Dado que en cada caso las funciones de utilidad del médico y del paciente responden a distintas variables, las ineficiencias en cada interacción resultaron ser diferentes según el médico actúe bajo una función salarial fija o variable.

A continuación, y luego de realizar un análisis comparativo, se determinan 
las potenciales herramientas regulatorias que podrían mejorar la conducta del médico en la atención del paciente en cada uno de los casos mencionados.

\section{Objetivos de las instituciones hospitalarias}

1. En el caso del hospital público el objetivo es maximizar el número de prestaciones dadas dos restricciones: una de tipo económica, el límite presupuestario (dado por el monto asignado por el Estado). La otra restricción es de orden físico dada por la infraestructura hospitalaria con que se dispone.

2. En el caso del hospital privado el objetivo es maximizar el beneficio y por ende el número de prestaciones que se llevan a cabo en pacientes con recursos o con algún tipo de seguro de salud, dadas dos restricciones: una de tipo ética, consistente en no generar prestaciones más allá de un cierto nivel que implique un desprestigio para la institución o para los profesionales que allí atienden, y la otra dada por las limitaciones fisicas impuestas por la infraestructura hospitalaria con que se dispone.

\section{Función salarial del médico como agente}

La función salarial del médico en el hospital público esta dada por un salario fijo definido como $w=\bar{w}$.

La función salarial del médico en el hospital privado surge de una función lineal del número de prestaciones, esto es $w=\bar{w}+k P$.

Ineficiencias en los tipos de esfuerzo

La conducta que adopte el médico, y por lo tanto la manipulación de su nivel de esfuerzo en la atención de sus pacientes, tiene en cada tipo de hospital relación directa con el tipo de salario que perciba por su trabajo.

Las ineficiencias resultantes son diferentes según el tipo de contrato:

1. en el caso del médico que recibe un salario fijo la ineficiencia se da en "la calidad" de la atención que le brinda a sus pacientes ya que su nivel de esfuerzo en dicha atención será el más bajo posible,

2. en el caso del médico que además recibe un salario en función de las prestaciones 
que realiza la ineficiencia se detecta en "la cantidad" de las prestaciones que le demanda a sus pacientes, dado el alto nivel de esfuerzo que le asigna a esta tarea.-

En ambos casos el resultado es ineficiente: en el primero por defecto y en el segundo por exceso. El médico del hospital público debería tener que realizar un mayor esfuerzo en la calidad de la atención pero realiza el más bajo posible mientras que el médico del hospital privado debería moderar su nivel de esfuerzo, pero adopta el nivel de esfuerzo más alto posible, lo que le permite maximizar sus ingresos inter-temporalmente.

Considerando los problemas tanto del sector público como del privado en términos de la relación entre nivel de esfuerzo y salario, puede verse que las ineficiencias descriptas están asociadas a dos puntos separados. Uno (indicado $c$ en el siguiente gráfico) corresponde al sector público en el que un salario fijo induce un nivel de esfuerzo bajo. En tanto el otro punto distinguido (d en el gráfico) corresponde al sector privado, en el que el pago salarial depende del número de prestaciones y conduce a un excesivo nivel de esfuerzo. Cualquier intento regulatorio deberia tender a incentivar un nivel de esfuerzo intermedio: mayor en el sector público y menor en el privado.

\section{Gráficamente:}

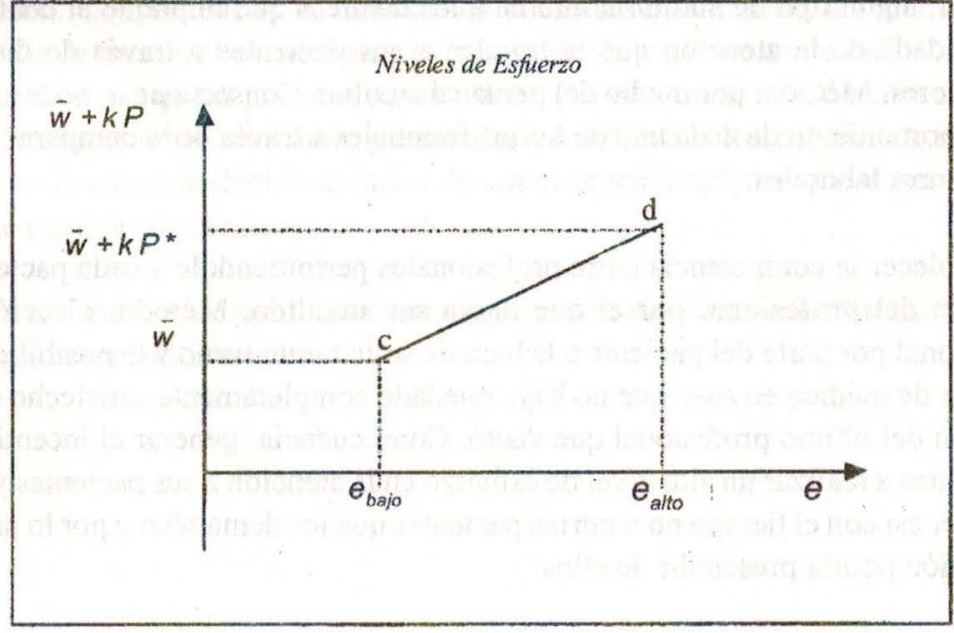




\section{Potencial regulación en el sector público}

Planteado el conflicto de intereses entre el médico y el paciente en relación al esfuerzo que realice el primero en la atención del segundo, resta plantear las herramientas regulatorias que puedan minimizar las ineficiencias discutidas. Dado que en nuestro caso de estudio el paciente no tiene en sus manos la posibilidad de diseñar el contrato de forma tal que genere en el médico los incentivos para realizar un esfuerzo óptimo, las posibles herramientas regulatorias deben ser impuestas desde el punto de vista institucional a partir de los contratos diseñados por los directores de hospitales con los profesionales de la salud de su institución. Estas herramientas regulatorias podrían ser:

1. Con respecto al salario, además de la remuneración fija que recibe el médico incorporar un "plus" a aquellos profesionales que "sólo" quieran trabajar en el sector público. Método: estimación del costo de oportunidad del profesional por resignar posiciones en el sector privado e incorporarlo como plus. Consecuencia: Se le incorpora en el contrato laboral un incentivo económico que reemplaza el ingreso percibido en el sector privado y genera las condiciones para que el médico tenga una mayor dedicación a su tarea dentro del hospital público resolviendo el conflicto del bajo nivel de esfuerzo.

2. Un sistema de información que controle para cada profesional el número de pacientes atendidos, el tiempo destinado a la atención de cada paciente, el porcentaje de derivación a otros servicios, el porcentaje de internaciones e intervenciones, etc. Es decir, algún tipo de auditoria interna a los médicos que implique el control de "la calidad" de la atención que le brindan a sus pacientes a través de diversos indicadores. Método: por medio del personal auxiliar. Consecuencia: poder inferir el comportamiento de cada uno de los profesionales a través de la comparación de indicadores laborales.

3. Establecer la competencia entre profesionales permitiéndole a cada paciente la elección del profesional por el que desea ser atendido. Método: elección del profesional por parte del paciente a la hora de solicitar un turno y la posibilidad de cambiar de médico en caso que no haya quedado completamente satisfecho con la atención del último profesional que visitó. Consecuencia: generar el incentivo en los agentes a realizar un alto nivel de esfuerzo en la atención a sus pacientes ya que de no ser así con el tiempo no tendrían pacientes que los demanden y por lo tanto la institución podría prescindir de ellos. 


\section{Potencial regulación en el sector privado}

Al igual que en el caso del hospital público se describen las potenciales herramientas regulatorias que desde el punto de vista institucional podrían mejorar la conducta de sus agentes:

1. La realización de auditorias externas por parte de las compañías aseguradoras (obras sociales y medicinas prepagas) a los médicos de modo de controlar el número de prácticas realizadas a sus afiliados como así también el porcentaje de derivación hacia otros servicios, el porcentaje de internaciones e intervenciones y todo tipo de indicadores, acerca de las actividades que realizan los médicos dentro del hospital y que impliquen un costo tanto al afiliado como a la compañía de seguros. Método: a través de médicos auditores que puedan corroborar la necesidad o no de las prestaciones realizadas a sus afiliados. Consecuencia: minimizar la potencial inducción de demanda por parte de los profesionales.

2. Con respecto al salario, las compañías aseguradoras (obras sociales y medicinas prepagas) podrian establecer un contrato con los médicos que implique un salario por capitación, es decir una remuneración fija por cada afiliado o beneficiario de la aseguradora. Método: el paciente elige su profesional de confianza y luego la aseguradora le transfiere al médico la cápita correspondiente a su paciente. Si el paciente no queda satisfecho con los servicios recibidos puede optar por otro profesional lo cuál introduce el factor de competencia que eliminará en el médico los incentivos a manipular las prestaciones demandadas y el nivel de esfuerzo en la atención. Consecuencia: la cantidad de consultas que implica la atención del paciente la determinará el médico, y la remuneración será constante más allá de la cantidad de veces que el paciente sea atendido. Por esta razón esta función salarial reduce los incentivos a la sobre-prestación e incentiva a los médicos a brindar calidad en la atención debido al factor de competencia implícito que le permite al paciente cambiar de médico.

En análisis desarrollado hasta aquí puede sintetizarse en un cuadro tal como aparece a continuación: 


\begin{tabular}{|c|c|c|}
\hline & HOSPITAL PUBLICO & HOSPITAL PRIVADO \\
\hline OBJETTVO & $\begin{array}{l}\text { Maximizar el Número de } \\
\text { Prestaciones }\end{array}$ & Maximizar el Beneficio \\
\hline $\begin{array}{l}\text { FINANCIAMIENTO } \\
\text { DE LAS } \\
\text { PRESTACIONES }\end{array}$ & $\begin{array}{l}\text { En Mayor Proporción } \\
\text { Recursos Públicos } \\
\text { En Menor Proporción } \\
\text { Obras Sociales Y } \\
\text { Medicinas Prepagas }\end{array}$ & $\begin{array}{l}\text { En Mayor Proporción } \\
\text { Recursos Privados } \\
\text { (Medicinas Prepagas, } \\
\text { Obras Sociales o } \\
\text { Dinero Circulante) }\end{array}$ \\
\hline $\begin{array}{c}\text { INTERNALIZACION } \\
\text { DE LOS COSTOS } \\
\text { POR PARTE DEL } \\
\text { PACIENTE }\end{array}$ & No & $\mathrm{Si}$ (en parte) \\
\hline $\begin{array}{c}\text { FUNCION } \\
\text { SALARIAL DEL } \\
\text { MEDICO }\end{array}$ & $\begin{array}{l}\text { Salario Fijo, } \\
w=\bar{w}\end{array}$ & $\begin{array}{l}\text { Salario Variable, } \\
w=\bar{w}+k P\end{array}$ \\
\hline INEFICIENCIAS & $\begin{array}{l}\text { Calidad de las } \\
\text { Prestaciones }\end{array}$ & Cantidad de Prestaciones \\
\hline $\begin{array}{c}\text { TIPO DE } \\
\text { ESFUERZO }\end{array}$ & Bajo & Alto \\
\hline $\begin{array}{l}\text { HERRAMIENTAS } \\
\text { REGULATORIAS }\end{array}$ & $\begin{array}{l}\text { - Salario } \\
\text { - Sistema de Información } \\
\text { - Auditorias Internas } \\
\text { - Competencia entre } \\
\text { Profesionales }\end{array}$ & $\begin{array}{l}\text { - Auditorias Externas } \\
\text { - Salario } \\
\text { - Competencia entre } \\
\text { Profesionales }\end{array}$ \\
\hline
\end{tabular}




\section{CONCLUSIONES}

Dada la asimetría de información entre el médico y el paciente se modeló esta realidad en términos de la relación agente - principal. Si bien coexisten diversas relaciones de agencia en el sector de atención de la salud, la relación médico paciente cobra especial relevancia debido a que el desempeño del médico determina a través de sus demandas derivadas un gran porcentaje del gasto total en salud.

El diseño del contrato laboral óptimo entre las instituciones hospitalarias y los médicos que en ellas ofrecen sus servicios implica considerar las funciones de preferencias tanto del médico como de los pacientes, además de las restricciones presupuestarias, físicas y humanas. Suponiendo que dicha información está disponible, cada director de hospital deberá conducir a través de sus contratos laborales, hacia una Función de Bienestar Social que logre acercar las preferencias de los médicos y de los pacientes hacia un estado de optimalidad en el sentido que no exista otro diseño de contratos (o mecanismo) que, con los recursos disponibles, mejore la situación de alguna de las partes sin que ninguna empeore.

En la actualidad los contratos laborales de los médicos en los hospitales públicos son mayoritariamente por un salario fijo mientras que en los hospitales privados el salario del médico tiene una componente variable que está en relación al número de prestaciones realizadas. Cada uno de estos contratos induce diferentes tipos de ineficiencias, el salario fijo no genera incentivos a realizar una atención de calidad provocando desempeños ineficientes con un tipo de esfuerzo bajo. El salario por prestación provee los incentivos para que el médico genere inducción de demanda y se garantice un mayor flujo de fondos.

El objetivo de las herramientas regulatorias propuestas, tanto en el sector público como en el privado, es generar los incentivos necesarios para situar el nivel de esfuerzo del médico entre el nivel bajo y el alto, es decir a lo largo del tramo cd de la función salarial expuesta en el gráfico de niveles de esfuerzo. De esta forma se obtendría un nivel de esfuerzo ineficiente con un tipo de esfuerzo bajo. El salario por prestación provee los incentivos para que el médico genere inducción de demanda y se garantice un mayor flujo de fondos.

Cada ineficiencia descripta plantea una diferente necesidad regulatoria si se pretende minimizarla. Sin embargo el control de la calidad en los hospitales públicos requiere una mayor cantidad de instrumentos regulatorios que los necesarios para el control de la cantidad en los hospitales privados. Mientras en el primer caso parecen ser necesarios controles en la función salarial, auditorias internas, sistemas 
de información y competencia entre profesionales, en el segundo caso la amenaza de las empresas o compañías aseguradoras (públicas o privadas) de excluir de sus convenios a los profesionales que abandonen el criterio ético en su desempeño parecen herramientas suficientes para optimizar los resultados. Es decir la competencia propia del sector privado permite alcanzar más fácilmente un resultado óptimo. Cuando la misma no existe, es necesario utilizar una mayor cantidad de herramientas correctoras que, además del costo implícito que generan, no garantizan alcanzar el resultado óptimo debido a la complejidad que implica la ejecución de las mismas.

Un dato relevante que merece ser considerado es el grado de información de los pacientes en cada tipo de institución. Los pacientes que acuden al sector público pertenecen, en general, al estrato de población de menores recursos y de menores posibilidades de alcanzar un mayor nivel educativo. Los pacientes que demandan su atención en el sector privado tienen, al menos, la posibilidad, a través de un seguro de salud (o de aportes monetarios), de realizar una inter-consulta $y$ disminuir el grado de incertidumbre con respecto a su estado de salud. Bajo este contexto, es aún más complejo medir la calidad de la atención recibida por la población con carencia informativa que la cantidad de prestaciones demandadas por los médicos en una población con mayor disponibilidad de información. Esto último adiciona complejidad al problema dado que no sólo resulta complicado medir la calidad desde la oferta sino también es difícil evaluarla desde la demanda.

Por fin resta un breve comentario acerca de cómo las formas de pago en cualquiera de las relaciones contractuales del sistema de atención de la salud pueden influenciar en el desempeño de otras partes del mismo. Un ejemplo de esto es el tipo de contrato con el que el gobierno le asigna el presupuesto a un hospital público. Las alternativas de elección entre un presupuesto prospectivo o un presupuesto de reembolso de costos implica un trade off de incentivos entre contención de costos y calidad. En el caso de un presupuesto prospectivo hay incentivos a llevar a cabo una producción técnicamente eficiente y por lo tanto, producir a costo mínimo. Sin embargo la debilidad de este tipo de contrato es que se seleccionen los buenos riesgos y se eluden los malos evitando los pacientes de alto costo. Esto último es la consecuencia de que el hospital debe internalizar los costos del tratamiento de sus pacientes y de que puede apropiarse de los remanentes del presupuesto no gastado. En el caso del presupuesto por reembolso de costos la fortaleza está dada por el incentivo a brindar un nivel de calidad óptimo mientras que la debilidad viene dada por la carencia de incentivos al ahorro en la cantidad de servicios prestados debido a que los costos reales de producción del hospital quedan totalmente cubiertos (Pere Ibern Regás, 1999).

Con este planteo es posible corroborar la influencia que ejercen las formas 
de pago en el desempeño de los diferentes actores del sistema de salud. Bajo los supuestos expuestos el desempeño de un médico en la atención de sus pacientes en un hospital público que opera bajo presupuesto prospectivo diferirá sustancialmente si el presupuesto es de reembolso por costos, y por lo tanto, no está sometido a una restricción presupuestaria.

Una de las futuras líneas de investigación a partir de este trabajo tendrá como eje central el análisis de relaciones de agencia común, en las que el médico no sólo es agente del paciente sino también del director del hospital. En este caso se internalizan no sólo los incentivos generados por el tipo de contrato laboral del médico, sino también las formas de pago que operan desde la órbita gubernamental hacia la institución hospitalaria. Esta discusión apunta a la necesidad de construir un modelo unificado en el que se traten los incentivos y el comportamiento de pacientes, médicos, hospitales y aseguradores simultáneamente.

\section{BIBLIOGRAFIA}

Bernheim, D. and Whinston, M., "Common Agency", Econométrica, Vol. 54, (4), July, 1986, p. 923-942.

Bertranou, F., "Health Care Services Utilization And Health Insurance Coverage: Evidence From Argentina", Department of Economics, University of Pittsburgh and Universidad Nacional de Cuyo, mimeo, 1998.

Bertranou, F., Delajara M. y Amiune, O., "Una Función de Producción de Salud Infantil para Argentina", Universidad Siglo 21, mimeo, Córdoba, 2002.

Corchón, L., The Theory of Implementation of Socially Optimal Decisions in 
Economics, Universidad de Alicante, The Macmillan Press LTD, Great Britain, 1996.

Díaz Muñoz, A. R., "Sistemas de Salud en Proceso de Reforma", OPS, OMS, Programa de políticas de salud, División de Salud y Desarrollo, Impresiones Avellaneda, Buenos Aires, 1994.

Dieter, B., "Pricing and Price Regulation: An Economic Theory for Public Enterprises and Public Utilities", Advanced Textbooks in Economics, (34), Amsterdam, Elsevier, 1994.

Donaldson, C.. and Gerard, K., Economics of Health Care Financing: The Visible Hand, The Macmillan Press LTD, Hong Kong, 1993.

Douglas, N., Instituciones, Cambio Institucional y Desempeño Económico, Fondo de Cultura Económica, México, 1993.

Eggertsson, T., El Comportamiento Económico y las Instituciones, Alianza Economía, Madrid,1995.

Feldman, R., Escribano, C. and Pellisé, L., "The Role of Government in Health Insurance Markets with Adverse Selection", Health Economics, 7: 659$670,1998$.

Feldstein, P. J., Health Care Economics, Delmar Publishers an International Thomson Publishing Company, Albany (N.Y.), 1999.

Flood, M. C. V., El Gasto Público Social y su Impacto Redistributivo, Caracteristicas de la evolución del Gasto Público Social, Ministerio de Economía y Obras y Servicios Públicos, Secretaría de Programación Económica, Buenos Aires, 1999.

Folland, S., Goodman, A. and Stano, M., The Economics of Health and Health Care, A Simon \& Schuster Company, second edition, Prentice Hall, New Jersey, 1997.

González García, G. y Tobar, F., Más Salud por el Mismo Dinero: La Reforma del Sistema de Salud en Argentina, Grupo Editor Latinoamericano, Buenos Aires, 1997.

Ibern Regás, P., Incentivos y Contratos en los Servicios de Salud, Springer-Verlag Ibérica, Barcelona, 1999.

Informe sobre Desarrollo Humano en la provincia de Buenos Aires, "Gasto en Salud y Resultados", Informes Sobre Desarrollo Humano en la Provincia de Buenos Aires, capitulo VII, 1999. http://bfdh.saij.jus.gov.ar/ datosfacticos/html/99all/bsas $99 . \mathrm{html}$

Katz, J. y Muñoz, A., “Organización del Sector Salud: Puja Distributiva y Equidad”, Comisión Económica Para América Latina y El Caribe, Buenos Aires, mimeo, 1988.

Lavila, M. C., "Inducción de Demanda. Metodologías", Tesis doctoral, Rovira - i Virgili, Reus, España.

Macho Stadler, I. y Pérez Castrillo,D., Introducción a la Economía de la Información, Editorial Ariel S.A., Barcelona, 1994. 
Madies, C., "Desregulación de Obras Sociales y Regulación de la Medicina Prepaga. Marco Regulatorio, Antecedentes y Proyectos", 2000. http:// www.isalud.com/investigacion/documentos.asp

Mas Collel, A. Whinston, M, and Green, J., Microeconomic Theory, Oxford University Press, New York, 1995.

Médici, A., "La Desregulación de las Obras Sociales. Un Episodio más de la Reforma de Salud en Argentina", Banco Interamericano de Desarrollo, Informe Técnico del Departamento de Desarrollo Sostenible, mimeo, 2002.

Ministerio de Economía y Ministerio de Salud: "Estimaciones del Gasto en Salud", Argentina, noviembre 2001. http://www.mecon.gov.ar

Organización Panamericana de la Salud, "Argentina Perfil del Sistema de Servicios de Salud", Programa de Organización y Gestión de Sistemas y Servicios de Salud, División de desarrollo de sistemas y servicios de Salud, 1ra. ed. 2 de noviembre de 1998, 2da. ed. 25 de febrero de 2001.http:// www.bvs.org.ar/pdf/perfil2001.pdf.

Phelps, Ch., "Diffusion of information un Medical Care", Journal of Economic Perspectives, vol. 6, (3), summer 1992, p. 23-42.

, Health Economics, second edition, Addison-Wesley Educational Publishers Inc., New York, 1997.

Rasmusen, E., Juegos e Información: Una Introducción a la Teoria de los Juegos, Fondo de Cultura Económica, México, 1996.

Reunión Regional Tripartita de la OrT con la colaboración de la Organización Panamericana de la Salud, El Gasto de Bolsillo en Salud en América Latina y El Caribe: Razones de Eficiencia para la Extensión de la Protección Social en Salud, México, 1999. http://www.oitopsmexico99.org.pe.

Sanhueza, R. y Ruiz Tagle, J., "Choosing Health Insurance in a Dual Health Care System: The Chilean Case", Department of Economics, University of Chile, mimeo, 2001.

Sapelli, C. y Vial, B., "Selección Adversa y Riesgo Moral en los Seguros de Salud en Chile: Relación Entre la Demanda por Prestaciones Médicas y el Seguro de Salud", Instituto de Economía, Pontificia Universidad Católica de Chile, mimeo, 2000.

Stiglitz, J., La Economía del Sector Público, segunda edición, Antoni Bosch editor, Barcelona, 1997.

Tafani, R., Privatización, Subcobertura y Reforma Competitiva en Salud, Publicaciones de la Universidad Nacional de Río Cuarto, agosto de 1996. , Economía Sanitaria, Publicaciones de la Universidad Nacional de Rio Cuarto, julio 2000.

Tobar, F., "El Futuro de la Salud en Argentina", 2000. http://www.isalud.com/ investigacion/documentos.asp

-, "Economía de la Reforma de los Seguros de Salud en Argentina", mimeo, julio 2001. http://www.paho.org/Spanish/HDP/HDD/tobar.pdf 
Torres, J. M., "The Importance of Genetic Services for the Theory of Health: A Basis for an Integrating View of Health", Centro de Lógica y Filosofia de la Ciencia. Universidad Nacional del Sur. Bahía Blanca, mimeo, 2002. 